ANNALES

POLONICI MATHEMATICI

$92.2(2007)$

\title{
A decomposition of complex Monge-Ampère measures
}

\author{
by YANG XING (Umeå)
}

\begin{abstract}
We prove a decomposition theorem for complex Monge-Ampère measures of plurisubharmonic functions in connection with their pluripolar sets.
\end{abstract}

1. Introduction. The purpose of this paper is to give a decomposition of complex Monge-Ampère measures associated to pluripolar sets of plurisubharmonic functions in the class $\mathcal{F}(\Omega)$ defined in [C1]. We denote by $\operatorname{PSH}(\Omega)$ the class of plurisubharmonic functions in a hyperconvex domain $\Omega$ and by $\mathrm{PSH}^{-}(\Omega)$ the subclass of negative functions. Recall that a set $\Omega \subset \mathbb{C}^{n}$ is said to be a hyperconvex domain if it is open, bounded, connected and there exists $\varrho \in \operatorname{PSH}^{-}(\Omega)$ such that $\{z \in \Omega ; \varrho(z)<-c\} \subset \subset \Omega$ for any $c>0$. The class $\mathcal{F}(\Omega)$ consists of all plurisubharmonic functions $u$ in $\Omega$ such that there exists a sequence $u_{j} \in \mathcal{E}_{0}(\Omega)$ with $u_{j} \searrow u$ as $j \rightarrow \infty$ and $\sup _{j} \int_{\Omega}\left(d d^{c} u_{j}\right)^{n}<\infty$, where $\mathcal{E}_{0}(\Omega)$ is the class of bounded plurisubharmonic functions $v$ with $\lim _{z \rightarrow \zeta} v(z)=0$ for all $\zeta \in \partial \Omega$ and $\int_{\Omega}\left(d d^{c} v\right)^{n}<\infty$. We also need the subclass $\mathcal{F}^{a}(\Omega)$ of functions from $\mathcal{F}(\Omega)$ whose Monge-Ampère measures put no mass on pluripolar subsets of $\Omega$. It is known that MongeAmpère measures $\left(d d^{c} u\right)^{n}$ for $u \in \mathcal{F}(\Omega)$ are well-defined finite measures in $\Omega$ (see [C1] for details).

Our main result is the following: Restriction of the complex MongeAmpère measure of a function $u \in \mathcal{F}(\Omega)$ onto its pluripolar set is still a Monge-Ampère measure of some function in $\mathcal{F}(\Omega)$. As an application we find that every Monge-Ampère measure of a function in $\mathcal{F}(\Omega)$ can be written as a sum of two Monge-Ampère measures, one of which has zero mass on any pluripolar set and the other is carried by the pluripolar set of the corresponding function.

It is a great pleasure for me to thank Urban Cegrell for many fruitful comments.

2000 Mathematics Subject Classification: Primary 32W20, 32U15.

Key words and phrases: complex Monge-Ampère operator, plurisubharmonic function. 
2. Theorems and proofs. We need an inequality.

Lemma ([X2]). Let $u, v \in \operatorname{PSH}(\Omega) \cap L^{\infty}(\Omega)$ be such that

$$
\liminf _{z \rightarrow \partial \Omega}(u(z)-v(z)) \geq 0 .
$$

Then for any $-1 \leq w \in \operatorname{PSH}^{-}(\Omega)$ we have

$$
(n !)^{-2} \int_{u<v}(v-u)^{n}\left(d d^{c} w\right)^{n}+\int_{u<v}(-w)\left(d d^{c} v\right)^{n} \leq \int_{u<v}(-w)\left(d d^{c} u\right)^{n} .
$$

Recall [X2] that a sequence $\left\{u_{j}\right\}$ of functions in $\operatorname{PSH}(\Omega)$ is said to be convergent in $C_{n}$ to a function $u$ on a subset $E$ of $\Omega$ if for any $\delta>0$ we have $C_{n}\left\{z \in E ;\left|u_{j}(z)-u(z)\right|>\delta\right\} \rightarrow 0$ as $j \rightarrow \infty$, where $C_{n}$ denotes the inner capacity introduced by Bedford and Taylor in [BT].

We denote by $\chi_{A}$ the characteristic function of the set $A$.

Theorem 1. Let $v \in \mathcal{F}(\Omega)$. Then there exists $u \in \mathcal{F}(\Omega)$ with $u \geq v$ in $\Omega$ such that

$$
\left(d d^{c} u\right)^{n}=\chi_{\{v=-\infty\}}\left(d d^{c} v\right)^{n} \quad \text { in } \Omega .
$$

Furthermore, let $g$ be the unique function in $\mathcal{F}^{a}(\Omega)$ with $\left(d d^{c} g\right)^{n}=$ $\chi_{\{v>-\infty\}}\left(d d^{c} v\right)^{n}$. Then $v \geq u+g$ in $\Omega$.

Proof. By Theorem 2.1 in [C1] we can take a sequence $v_{j} \in \mathcal{E}_{0}(\Omega)$ such that $v_{j} \searrow v$ as $j \rightarrow \infty$. By [C2], [K] there exist $u_{j}^{k} \in \mathcal{E}_{0}(\Omega)$ such that $\left(d d^{c} u_{j}^{k}\right)^{n}=-\max (v / k,-1)\left(d d^{c} v_{j}\right)^{n}$. From the comparison theorem [BT] it follows that $u_{j}^{k+1} \geq u_{j}^{k} \geq v_{j} \geq v$. By passing to a subsequence if necessary, we assume that $u_{j}^{k} \rightarrow u^{k} \in \mathcal{F}(\Omega)$ weakly as $j \rightarrow \infty$, and $u^{k} \nearrow u \in \mathcal{F}(\Omega)$ as $k \rightarrow \infty$. Then Theorem 2 below shows that $\left(d d^{c} u^{k}\right)^{n}=$ $-\max (v / k,-1)\left(d d^{c} v\right)^{n}$, which implies $\left(d d^{c} u\right)^{n}=\chi_{\{v=-\infty\}}\left(d d^{c} v\right)^{n}$. If furthermore $\chi_{\{v>-\infty\}}\left(d d^{c} v\right)^{n}=\left(d d^{c} g\right)^{n}$ for $g \in \mathcal{F}^{a}(\Omega)$, then we take $g_{j}^{k} \in \mathcal{E}_{0}(\Omega)$ such that

$$
\begin{aligned}
\left(d d^{c} g_{j}^{k}\right)^{n} & =\max ((v+k) / k, 0)\left(d d^{c} v_{j}\right)^{n} \\
& =\max ((v+k) / k, 0)\left(d d^{c} \max \left(v_{j},-k-1\right)\right)^{n} .
\end{aligned}
$$

By the comparison theorem [BT] we have $0>g_{j}^{k} \geq \max \left(v_{j},-k-1\right) \geq v$. By Theorem 2 again, we assume that $g_{j}^{k}$ converges to a bounded psh function $g^{k}$ in $C_{n}$ on each $E \subset \subset \Omega$. Letting $j \rightarrow \infty$ we get $\left(d d^{c} g^{k}\right)^{n}=\max ((v+k) / k, 0)\left(d d^{c} v\right)^{n}=\max ((v+k) / k, 0)\left(d d^{c} g\right)^{n} \leq\left(d d^{c} g\right)^{n}$, which implies $0>g^{k} \geq g$. Hence $g^{k}$ decreases to some $g_{1} \in \mathcal{F}^{a}(\Omega)$. By Theorem 5.15 in [C1] we have $g_{1}=g$. Since $\left(d d^{c}\left(g_{j}^{k}+u_{j}^{k}\right)\right)^{n} \geq\left(d d^{c} g_{j}^{k}\right)^{n}+$ $\left(d d^{c} u_{j}^{k}\right)^{n}=\left(d d^{c} v_{j}\right)^{n}$ we get $v_{j} \geq g_{j}^{k}+u_{j}^{k}$ and hence $v \geq g+u$. The proof of Theorem 1 is complete. 
Theorem 2. Suppose that $v \in \mathcal{F}(\Omega), v_{j} \in \mathcal{E}_{0}(\Omega)$ and $-1 \leq \psi \in$ $\operatorname{PSH}^{-}(\Omega)$ are such that $v_{j} \searrow v$ as $j \rightarrow \infty$ and $v$ is bounded on $\{z \in$ $\Omega ; \psi(z) \neq-1\}$. If $u_{j} \in \mathcal{E}_{0}(\Omega)$ are such that $\left(d d^{c} u_{j}\right)^{n}=-\psi\left(d d^{c} v_{j}\right)^{n}$ and $u_{j} \rightarrow u \in \operatorname{PSH}(\Omega)$ weakly in $\Omega$, then $\left(d d^{c} u\right)^{n}=-\psi\left(d d^{c} v\right)^{n}, u \geq v$ and hence $u \in \mathcal{F}(\Omega)$.

Proof. By the comparison theorem [BT] we get $0 \geq u_{j} \geq v_{j} \geq v$. Hence $u \geq v$ and $u \in \mathcal{F}(\Omega)$. To prove $\left(d d^{c} u\right)^{n}=-\psi\left(d d^{c} v\right)^{n}$, by Theorem 7 in [X1] or [C1] we have $-\psi\left(d d^{c} v_{j}\right)^{n} \rightarrow-\psi\left(d d^{c} v\right)^{n}$ weakly as $j \rightarrow \infty$, and hence it is enough to show that $u_{j} \rightarrow u$ in $C_{n}$ on each $E \subset \subset \Omega$ as $j \rightarrow \infty$. Take $t<\inf _{\{\psi \neq-1\}} v$. Since

$$
\begin{aligned}
\left(d d^{c} v_{j}\right)^{n} & =\chi_{\left\{v_{j}>t\right\}}\left(d d^{c} v_{j}\right)^{n}+\chi_{\left\{v_{j} \leq t\right\}}\left(d d^{c} v_{j}\right)^{n} \\
& \leq\left(d d^{c} \max \left(v_{j}, t\right)\right)^{n}+\left(d d^{c} u_{j}\right)^{n} \leq\left(d d^{c}\left(\max \left(v_{j}, t\right)+u_{j}\right)\right)^{n},
\end{aligned}
$$

we have $v_{j} \geq u_{j}+\max \left(v_{j}, t\right)$ and thus $v \geq u+t$. Given $E \subset \subset \Omega$ and $0<\varepsilon<-t$, Theorem 6.10 of [BT] shows that there exists $0<\delta<1$ such that $C_{n}\{z \in E ;(1-\delta) v \leq-\varepsilon\}<\varepsilon$. By quasicontinuity of psh functions and Hartogs' lemma, we only need to show that

$$
C_{n}\left\{z \in E ; u(z)>u_{j}(z)+3 \varepsilon\right\} \rightarrow 0 \quad \text { as } j \rightarrow \infty .
$$

Let $l_{j}:=\min _{\Omega}\left(\delta u_{j}+\varepsilon\right)$. Since $C_{n}\left\{z \in E ; u_{j}(z) \leq \delta u_{j}(z)-\varepsilon\right\} \leq C_{n}\{z \in E$; $(1-\delta) v \leq-\varepsilon\}<\varepsilon$, we have

$$
C_{n}\left\{z \in E ; u(z)>u_{j}(z)+3 \varepsilon\right\} \leq C_{n}\left\{z \in \Omega ; u(z)>\delta u_{j}(z)+2 \varepsilon\right\}+\varepsilon,
$$

which, by the definition of $C_{n}$, does not exceed

$$
\begin{array}{r}
\sup \left\{\frac{1}{\varepsilon^{n}} \int_{u>\delta u_{j}+\varepsilon}\left(u-\delta u_{j}-\varepsilon\right)^{n}\left(d d^{c} w\right)^{n} ; w \in \operatorname{PSH}(\Omega), 0<w<1\right\}+\varepsilon \\
=\sup \left\{\frac{1}{\varepsilon^{n}} \int_{\max \left(u, l_{j}\right)>\delta u_{j}+\varepsilon}\left(\max \left(u, l_{j}\right)-\delta u_{j}-\varepsilon\right)^{n}\left(d d^{c} w\right)^{n} ;\right. \\
w \in \operatorname{PSH}(\Omega), 0<w<1\}+\varepsilon,
\end{array}
$$

which by the Lemma is less than

$$
\begin{aligned}
\frac{(n !)^{2} \delta^{n}}{\varepsilon^{n}} \int_{\max \left(u, l_{j}\right)>\delta u_{j}+\varepsilon}\left(d d^{c} u_{j}\right)^{n}+\varepsilon & \leq \frac{(n !)^{2} \delta^{n}}{\varepsilon^{n}} \int_{u>\delta u_{j}+\varepsilon}\left(d d^{c} v_{j}\right)^{n}+\varepsilon \\
& \leq \frac{(n !)^{2} \delta^{n}}{\varepsilon^{n}} \int_{u>\delta u_{j}+\varepsilon} \phi\left(d d^{c} v_{j}\right)^{n}+2 \varepsilon
\end{aligned}
$$

for some $\phi \in C_{0}^{\infty}(\Omega)$ with $0 \leq \phi \leq 1$, where we have used the fact that there exists $E_{1} \subset \subset \Omega$ such that $\int_{\Omega \backslash E_{1}}\left(d d^{c} v_{j}\right)^{n} \leq \varepsilon^{n+1} /(n !)^{2} \delta^{n}$ for all $j$, which follows from $\left(d d^{c} v_{j}\right)^{n} \rightarrow\left(d d^{c} v\right)^{n}$ weakly and $\lim _{j \rightarrow \infty} \int_{\Omega}\left(d d^{c} v_{j}\right)^{n}=$ $\int_{\Omega}\left(d d^{c} v\right)^{n}<\infty$. Since $v-t \geq u \geq v$ and $u_{j} \geq v$, we have $\left\{u>\delta u_{j}+\varepsilon\right\} \subset$ 
$\{v>a\}$ for $a:=(\varepsilon+t) /(1-\delta)<0$. So the last integral equals

$$
\begin{aligned}
& \quad \int_{\max (u, a)>\delta \max \left(u_{j}, a\right)+\varepsilon} \phi\left(d d^{c} v_{j}\right)^{n} \\
& \leq \frac{1}{\varepsilon} \int_{\max (u, a)>\delta \max \left(u_{j}, a\right)+\varepsilon} \phi\left(\max (u, a)-\max \left(u_{j}, a\right)\right)\left(d d^{c} v_{j}\right)^{n} .
\end{aligned}
$$

Since $v_{j} \geq u_{j}+t$ and $v_{j} \geq v \geq u+t$ we have $\max (u, a)-\max \left(u_{j}, a\right)=0$ if $v_{j} \leq a+t$. By the quasicontinuity of $u$ there exists an open subset $O_{\varepsilon} \subset \Omega$ such that $C_{n}\left(O_{\varepsilon}\right)<\varepsilon^{n+2}$ and $u \in C\left(\Omega \backslash O_{\varepsilon}\right)$. It then follows from Hartogs' lemma that $\varepsilon^{n+2}+\max (u, a) \geq \max \left(u_{j}, a\right)$ on $\operatorname{supp} \phi \backslash O_{\varepsilon}$ for all $j$ large enough. Hence by the definition of $C_{n}$, for all $j$ large enough we have

$$
\begin{aligned}
C_{n}\{z \in E & \left.; u(z)>u_{j}(z)+3 \varepsilon\right\} \\
\leq & \frac{(n !)^{2} \delta^{n}}{\varepsilon^{n+1}} \int_{\Omega} \phi\left(\varepsilon^{n+2}+\max (u, a)-\max \left(u_{j}, a\right)\right)\left(d d^{c} v_{j}\right)^{n} \\
& +2 \varepsilon+\varepsilon(n !)^{2}\left(\varepsilon^{n+2}-a\right)(-a-t)^{n} \sup _{\Omega}|\phi| \\
= & \frac{(n !)^{2} \delta^{n}}{\varepsilon^{n+1}} \int_{\Omega} \phi\left(\max (u, a)-\max \left(u_{j}, a\right)\right)\left(\left(d d^{c} \max \left(v_{j}, a+t\right)\right)^{n}\right. \\
& \left.+\frac{(n !)^{2} \delta^{n}}{\varepsilon^{n+1}} \int_{\Omega} \phi\left(\max (u, a)-\max \left(u_{j}, a\right)\right)\left(d d^{c} \max (v, a+t)\right)^{n}\right) \\
= & \mathrm{O}(\varepsilon) \quad \text { as } j \rightarrow \infty,
\end{aligned}
$$

where the last estimate follows from Theorem 1 and Corollary 1 in [X1] or [C2]. By the arbitrariness of $\varepsilon>0$ we see that $u_{j} \rightarrow u$ in $C_{n}$ on $E$ as $j \rightarrow \infty$, which concludes the proof of Theorem 2 .

Corollary 1. A positive measure $\mu$ in $\Omega$ can be written as $\mu=\left(d d^{c} v\right)^{n}$ for $v \in \mathcal{F}(\Omega)$ if and only if

$$
\mu=\left(d d^{c} u_{1}\right)^{n}+\chi_{\left\{u_{2}=-\infty\right\}}\left(d d^{c} u_{2}\right)^{n}
$$

for some $u_{1} \in \mathcal{F}^{a}(\Omega)$ and $u_{2} \in \mathcal{F}(\Omega)$.

Proof. The "only if" part. By $[\mathrm{C} 2],[\mathrm{K}]$ there exists a decreasing sequence $g_{k} \in \mathcal{E}_{0}(\Omega)$ such that $g_{k} \geq v$ in $\Omega$ and $\left(d d^{c} g_{k}\right)^{n}=\chi_{\{v>-k\}}\left(d d^{c} v\right)^{n}$. Then $u_{1}:=\lim _{k \rightarrow \infty} g_{k} \in \mathcal{F}^{a}(\Omega)$ and $\left(d d^{c} u_{1}\right)^{n}=\chi_{\{v \neq-\infty\}}\left(d d^{c} v\right)^{n}$. Hence we have $\mu=\left(d d^{c} u_{1}\right)^{n}+\chi_{\{v=-\infty\}}\left(d d^{c} v\right)^{n}$.

The "if" part. From Theorem 1 it turns out that there exists $h \in \mathcal{F}(\Omega)$ such that $\mu=\left(d d^{c} u_{1}\right)^{n}+\left(d d^{c} h\right)^{n}$. By Theorem 5.11 in [C1] there exist $\psi \in \mathcal{E}_{0}(\Omega)$ and $f \in L_{\mathrm{loc}}\left(\left(d d^{c} \psi\right)^{n}\right)$ such that $\left(d d^{c} u_{1}\right)^{n}=f\left(d d^{c} \psi\right)^{n}$. Take a sequence $h_{j} \in \mathcal{E}_{0}(\Omega)$ such that $h_{j} \searrow h$ as $j \rightarrow \infty$. Since $\min \left(f, k^{n}\right)\left(d d^{c} \psi\right)^{n}+$ 
$\left(d d^{c} h_{j}\right)^{n} \leq\left(d d^{c}\left(k \psi+h_{j}\right)\right)^{n}$, by [C2], [K] there exist $v_{j}^{k} \in \mathcal{E}_{0}(\Omega)$ such that $\left(d d^{c} v_{j}^{k}\right)^{n}=\min \left(f, k^{n}\right)\left(d d^{c} \psi\right)^{n}+\left(d d^{c} h_{j}\right)^{n}$ and hence the comparison theorems in [BT], [C1] imply that $0>v_{j}^{k} \geq k \psi+h \geq u_{1}+h$. Repeating the proof of Theorem 2 we obtain an increasing sequence $v^{k}$ in $\mathcal{F}(\Omega)$ such that $\left(d d^{c} v^{k}\right)^{n}=\min \left(f, k^{n}\right)\left(d d^{c} \psi\right)^{n}+\left(d d^{c} h\right)^{n}$ and $0>v^{k} \geq u_{1}+h$. Therefore, $v:=\left(\lim _{k \rightarrow \infty} v^{k}\right)^{*} \in \mathcal{F}(\Omega)$ and $\mu=\left(d d^{c} v\right)^{n}$. The proof of Corollary 1 is complete.

COROLlary 2. For any set $B=\left\{z_{1}, \ldots, z_{m}\right\}$ of points in $\Omega$ and nonnegative constants $c_{1}, \ldots, c_{m}$ there exists a function $u \in \operatorname{PSH}(\Omega) \cap L_{\mathrm{loc}}^{\infty}(\Omega \backslash B)$ such that $u=0$ on $\partial \Omega$ and $\left(d d^{c} u\right)^{n}=\sum_{j=1}^{m} c_{j} \delta_{z_{j}}$ in $\Omega$, where $\delta_{z_{j}}$ denotes the Dirac measure at $z_{j}$.

Proof. Take the pluricomplex Green function $g_{z_{j}}$ of $\Omega$ with logarithmic pole at $z_{j}$ and set $v=\sum_{j=1}^{m} c_{j}^{1 / n} g_{z_{j}}$. Then $v \in \mathcal{F}(\Omega) \cap L_{\text {loc }}^{\infty}(\Omega \backslash B)$ and $v=0$ on $\partial \Omega$. By Lemma 5 in [X3], $\left(d d^{c} v\right)^{n}$ has zero mass at any point $z \notin B$ and has mass $c_{j}$ at $z_{j}$. Therefore, by Theorem 1 we get the required function $u$ and the proof is complete.

\section{References}

[BT] E. Bedford and B. A. Taylor, A new capacity for plurisubharmonic functions, Acta Math. 149 (1982), 1-40.

[C1] U. Cegrell, The general definition of the complex Monge-Ampère operator, Ann. Inst. Fourier (Grenoble) 54 (2004), 159-197.

[C2] - Pluricomplex energy, Acta Math. 180 (1998), 187-217.

[K] S. Kołodziej, The range of the complex Monge-Ampère operator, II, Indiana Univ. Math. J. 44 (1995), 765-782.

[X1] Y. Xing, Convergence in capacity, Umeå Univ., Research Reports No 1, 2007.

[X2] - Continuity of the complex Monge-Ampère operator, Proc. Amer. Math. Soc. 124 (1996), 457-467.

[X3] - , The complex Monge-Ampère equations with a countable number of singular points, Indiana Univ. Math. J. 48 (1999), 749-765.

Department of Mathematics

University of Umeå

S-901 87 Umeå, Sweden

E-mail: Yang.Xing@mathdept.umu.se

Received 8.3.2007

and in final form 19.4.2007 